\section{BRAZIULIAN JOURNAL}

OF MEDICAL AND BIOLOGICAL RESHARCH

www.bjournal.com.br
ISSN 0100-879X

Volume 44 (6) 497-605 June 2011

BIOMEDICAL SCIENCES

AND

CLINICAL INVESTIGATION

Braz J Med Biol Res, June 2011, Volume 44(6) 497

doi: 10.1590/S0100-879X2011000600001

Cell therapy in dilated cardiomyopathy - back to the right scientific track? Comment on "Cell therapy in dilated cardiomyopathy: from animal models to clinical trials" [Braz J Med Biol Res 2011; 44: 388-393]

\title{
J.A. Marin-Neto
}

The Brazilian Journal of Medical and Biological Research is partially financed by

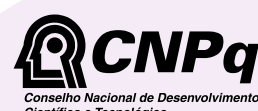

da Ciência e Tecnologia

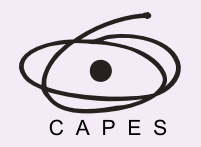

Ministério da Educação

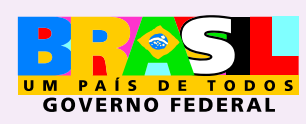

\section{JAPESP}

Institutional Sponsors
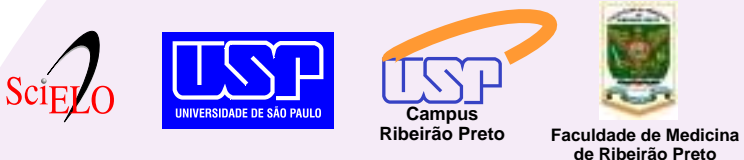

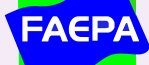

e Ribeirão Preto

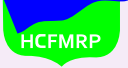

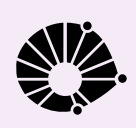

Ф SHIMADZU

GE Healthcare
Hotsite of proteomics metabolomics developped by:

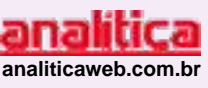

Thermo SCIENTIFIC 


\section{Cell therapy in dilated cardiomyopathy: back to the right scientific track? \\ Comment on "Cell therapy in dilated cardiomyopathy: from animal models to clinical trials" [Braz J Med Biol Res 2011; 44: 388-393]}

Divisão de Cardiologia, Departamento de Clínica Médica, Faculdade de Medicina de Ribeirão Preto, Universidade de São Paulo, Ribeirão Preto, SP, Brasil

This is a timely review of cell therapies, which have been tested in animal models of dilated cardiomyopathy induced by a variety of chemical and infectious stimuli including genetic manipulation of the host. The authors also provide some insight on the few published or ongoing investigations concerning autologous cell therapy in humans with dilated cardiomyopathy.

The picture that emerges from the research carried out on experimental animals is mainly characterized by an enormous heterogeneity of animal species (rats, mice, hamsters, dogs); methods of inducing dilated cardiomyopathy (genetic engineering, toxic and other harmful agents, rapid pacing of the heart); cell types used (embryo-derived stem cells, other stem cells, fully differentiated cardiomyocytes, skeletal myoblasts, endothelial progenitor cells, skeletal myoblasts); techniques of injecting them into the diseased heart (intracoronary, intramyocardial); use of adjuvant factors (hepatocyte growth factor, mesenchymal-derived factor); methods for the evaluation of the effects of the experimental procedures or for their interpretation (engrafting of injected cells into the heart, differentiation of injected cells into cardiomyocytes, vascular endothelial and smooth muscle cells, secretion of angiogenic, antiapoptotic and mitogenic factors, improvement in functional mechanical properties of the whole heart such as left ventricular ejection fraction).

From such a striking diversity of investigational approaches, it is no wonder that the reported results have been so variable. It is also clear that the ultimate goals of these studies, i.e., to achieve cardioprotective effects and/or to regenerate components of the diseased heart, remain quite elusive. Despite this, the Authors of the review concluded that the results from the experimental animal models were encouraging enough to stimulate the application of stem cell therapy to humans with dilated cardiomyopathy.
Research regarding cell therapy in humans is hindered by ethical and regulatory issues and by restrictions to the use of some cell types, including logistic problems and the possibility of untoward side effects such as the generation of teratomas. Similarly to research in experimental animals, the current scenario in human investigation of cell therapy for non-ischemic dilated cardiomyopathy as depicted in the review by del Corsso and Campos de Carvalho is also full of uncertainties. Although the procedures have been generally reported to be safe, this conclusion may be actually unwarranted because the long-term effects of cell therapy in humans have not been adequately described. For instance, even the balloon inflation technique that is recommended during the intracoronary injection of cells has the potential of damaging the coronary endothelium and may elicit a delayed proliferative response to trauma that could lead to local stenosis.

Reported results are also usually open to question, reflecting the methodological flaws that pervade most of the studies. The described changes in symptoms and in individual values of left ventricular ejection fraction most likely represent random fluctuations during the natural history of the cardiomyopathy and/or the effect of uncontrolled factors, such as change in concomitant pharmacological therapies. It is quite disturbing to see that a change in the study endpoint of left ventricular ejection fraction seen when one method was used was not corroborated when another method was employed in the same group of patients.

On the basis of these considerations, it would seem wise not to embark into further cell therapy studies in humans suffering from such a complex entity as dilated cardiomyopathy before the investigation of animal models of the disease is placed in the right scientific perspective by more appropriately designed and conducted experimental studies.

Correspondence: J.A. Marin-Neto, Divisão de Cardiologia, Departamento de Clínica Médica, Faculdade de Medicina de Ribeirão Preto, USP, Av. Bandeirantes, 3900, 14049-900 Ribeirão Preto, SP, Brasil. E-mail: jamneto@fmrp.usp.br

Received April 15, 2011. Accepted April 30, 2011. Available online June 13, 2011. Published June 13, 2011. 